\title{
Prostate-Specific Membrane Antigen-Guided Surgery
}

\author{
Tobias Maurer ${ }^{1,2}$, Markus Graefen ${ }^{1}$, Henk van der Poel ${ }^{3}$, Freddie Hamdy ${ }^{4}$, Alberto Briganti ${ }^{5}$, Matthias Eiber ${ }^{6}$, \\ Hans-Jürgen Wester ${ }^{7}$, and Fijs W.B. van Leeuwen ${ }^{3,8}$ \\ ${ }^{I}$ Martini-Klinik Prostate Cancer Center, University Hospital Hamburg-Eppendorf, Hamburg, Germany; ${ }^{2}$ Department of Urology, \\ University Hospital Hamburg-Eppendorf, Hamburg, Germany; ${ }^{3}$ Department of Urology, Antoni van Leeuwenhoek Hospital-The \\ Netherlands Cancer Institute, Amsterdam, The Netherlands; ${ }^{4}$ Nuffield Department of Surgical Sciences, University of Oxford, \\ Oxford, United Kingdom; ${ }^{5}$ Department of Urology, Division of Oncology, Urological Research Institute, IRCCS Ospedale San \\ Raffaele, Milan, Italy; ${ }^{6}$ Department of Nuclear Medicine, Technical University of Munich, Munich, Germany; ${ }^{7}$ Pharmaceutical \\ Radiochemistry, Technical University of Munich, Munich, Germany; and ${ }^{8}$ Interventional Molecular Imaging Laboratory, Department \\ of Radiology, Leiden University Medical Centre, Leiden, The Netherlands
}

Since its introduction to the diagnostic pathway for prostate cancer management, prostate-specific membrane antigen (PSMA)-ligand PET has demonstrated great potential. PSMA-ligand imaging is increasingly influencing therapeutic decision making, although its impact on patient outcomes still needs to be defined. One relatively new application, enabled through chemical and engineering efforts, is PSMA-guided surgery. This review highlights the potential of PSMA-guided surgery and discusses its implications in lymph node dissection in primary and recurrent prostate cancer.

Key Words: prostate cancer; image-guided surgery; salvage; lymph node dissection; fluorescence

J Nucl Med 2020; 61:6-12

DOI: 10.2967/jnumed.119.232330

$\mathbf{T}$ he lymph nodes represent one of the sites most frequently affected by recurrent prostate cancer (PC). Recognizing the exact state and extent of lymphatic spread represents a complex challenge. Besides the application of several clinical variables such as histologic type from biopsy or previous surgery, prostate-specific antigen (PSA) value, and PSA velocity and nomograms, application of sensitive molecular imaging can substantially move the field forward.

Nodal tumor infiltration can be considered as locoregional progressive disease or as a state between localized disease on one side and, on the other side, oligometastatic disease in which a few (commonly less than 3-5) metastatic lesions are located outside the pelvic lymph nodes or systemic metastatic disease. Patients with early locoregional lymphatic metastatic disease exhibit a better prognosis. As a result, locoregional lymphatic metastatic disease should be distinguished from the oligometastatic disease at other sites (e.g., bone), which is more closely related to systemic metastatic disease (1). Thus, localized treatment in this early state of metastasis might yield even better oncologic control than local treatment of oligometastatic disease.

Received Sep. 16, 2019; revision accepted Oct. 22, 2019.

For correspondence or reprints contact: Tobias Maurer, Martini-Klinik Prostate Cancer Center, University Hospital Hamburg-Eppendorf, Martinistrasse 52 Gebäude Ost 46, 20246 Hamburg, Germany.

E-mail: t.maurer@uke.de

Published online Nov. 15, 2019.

COPYRIGHT (C) 2020 by the Society of Nuclear Medicine and Molecular Imaging.
On progression of local PC, cancer cells hijack the lymphatics originating from the prostate itself or surrounding tissues. In the lymphatics, these cells travel to draining lymph nodes. Within the lymphatic sinus, cancer cells can reside, multiply, and form tumor satellites that progress from micro- to macrometastases (usually defined as tumor deposits $\geq 2 \mathrm{~mm}$ ) (2).

In primary $\mathrm{PC}$, natural local lymphatic drainage is preserved. Nevertheless, lymphatic drainage may vary substantially between individuals. As a result, in a significant proportion of patients, metastatic lymph nodes are also found outside the classic template of an extended pelvic lymph node dissection (ePLND) (3). Additionally, there are reports of tumor-based blockages in lymphatic ducts or lymph nodes, which could induce a diversion of conventional lymphatic dissemination pathways (4). Therapeutic interventions such as radical prostatectomy or radiation therapy may significantly impact lymphatic drainage pathways. For this reason, metastases in recurrent PC may occur in unexpected locations. These metastases are thought to arise from cancer cells that escaped the tumor before radical prostatectomy and have resided in the surrounding tissue or lymphatics or, in the case of primary radiation therapy, might also spread from the prostate itself. To recognize the exact state and extent of disease in recurrent PC represents a great challenge and remains complex but significantly impacts proposed treatment (Fig. 1). Besides the use of several clinical variables such as primary histology, serum PSA, and PSA kinetics, the field of molecular PET imaging has moved forward substantially with the use of choline-based tracers and, especially, tracers directed against the prostate-specific membrane antigen (PSMA) (5-7).

\section{PSMA-LIGAND PET IMAGING}

PSMA, albeit not as specific as its name suggests, shows a significant overexpression (100- to 1,000-fold) on most PC cells $(>90 \%)(8,9)$. Within the last decade, several small-molecule radiotracers have been developed for PET imaging for staging and restaging of PC (10). Today, there is no clear evidence of the superiority of one of the clinically available radiotracers. PSMAligand PET is able to visualize metastatic lesions both at low PSA values (11-15) and at small sizes (16). Similar to other imaging techniques, detection efficacy decreases with lesion size in PSMAligand PET. Additionally, detection is directly linked to the magnitude of PSMA overexpression in a metastasis. In a meticulous analysis in recurrent-PC patients undergoing salvage lymph node 


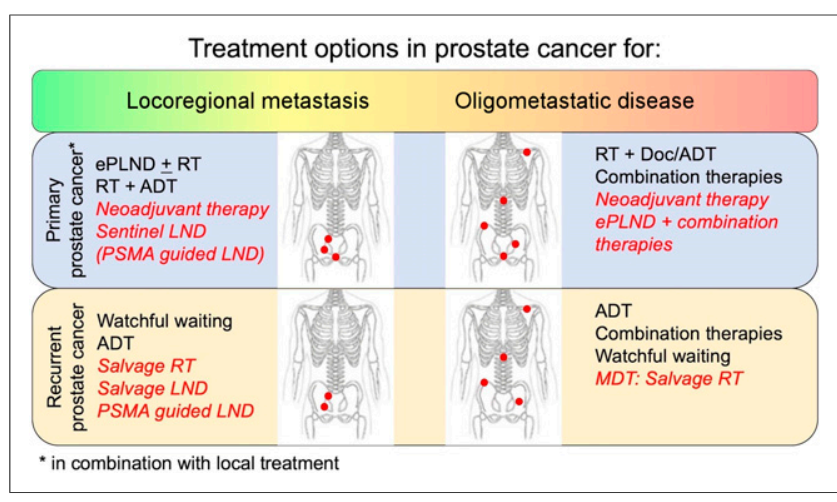

FIGURE 1. Treatment options for locoregional or oligometastatic primary and recurrent prostate cancer on advanced molecular imaging. Standard options are in black font; experimental approaches are in red font. $A D T=$ androgen deprivation therapy; Doc $=$ docetaxel; $L N D=$ lymph node dissection; MDT = metastasis-directed therapy; RP = radical prostatectomy; RT = radiotherapy.

dissection (sLND), PSMA-ligand PET detection rates for metastases were more than $50 \%$ and $90 \%$ when the short-axis diameter of the metastatic lesion was more than 2.3 and $4.5 \mathrm{~mm}$, respectively (17). It can be expected that the same holds true for primary lymph node metastases. Also, the travel pathway of positron emissions in tissue limits the intrinsic spatial resolution of clinical PET imaging and is dependent on the nuclide used (e.g., ${ }^{68} \mathrm{Ga}$ or ${ }^{18} \mathrm{~F}$ ). As a result, micrometastases $(<2 \mathrm{~mm})$ cannot be accurately detected by PSMA-ligand PET. Nevertheless, PSMA-ligand PET clearly outperforms conventional CT or MRI, which detect lymph node metastases only if their size exceeds $8-10 \mathrm{~mm}(18,19)$. Only lymphatic surgical mapping technologies provide an alternative for the identification of micrometastases smaller than $2 \mathrm{~mm}$.

Currently, there is still no evidence that clinical decisions based on PSMA-ligand PET improve oncologic outcomes in the management of PC (20). As a result, several guidelines, such as the guideline of the European Association of Urology, do not explicitly recommend or disregard PSMA-ligand PET in the primary staging setting. Nevertheless, PSMA-ligand PET is increasingly being used

\section{NOTEWORTHY}

- The superior predictive diagnostic accuracy of PSMA-ligand PET for metastatic prostate cancer lesions (e.g., lymph nodes) has provided a basis for PSMA-targeted surgical resections.

- For nodal staging or management of local lymphatic metastases, ePLND is currently considered the standard approach.

- Radioactive labeling of sentinel lymph nodes in primary PC and PSMA-based targeting of metastases in recurrent PC intends to increase diagnostic and therapeutic results.

- Technical developments in tracer design (e.g., fluorescent PSMA ligands and hybrid ${ }^{99 \mathrm{~m}} \mathrm{Tc}$ /fluorescent PSMA ligands combining both approaches), as well as hardware developments (e.g., drop-in $\gamma$-probes for minimally invasive robotic surgery), are likely to advance the field of image-guided urology.

- The oncologic benefit of and indication for PSMA-targeted approaches still have to be more precisely defined. and evaluated in high-risk primary-PC patients. In patients with recurrent $\mathrm{PC}$, however, several guidelines changed recently, and the most recent European Association of Urology guideline advocates the use of PSMA-ligand PET after radical prostatectomy when the PSA level is higher than $0.2 \mathrm{ng} / \mathrm{mL}$ and when the results will influence subsequent treatment decisions (level IIb evidence, weak recommendation) (20). In addition, PSMA-ligand PET is recommended as the primary imaging modality in biochemical recurrence after primary curative radiation therapy (level IIb evidence, strong recommendation).

\section{PSMA-GUIDED SURGERY AS MOLECULARLY TARGETED PRECISION SURGERY}

In patients with biochemical recurrence after primary curativeintent radical prostatectomy, salvage external-beam radiotherapy is recommended when a local recurrence is suspected in the absence of detectable distant metastases. Equally, salvage prostatectomy might be considered as a potentially curative therapy option in selected cases of local recurrence after primary radiotherapy or ablative treatment. In cases of distant recurrence, or failure after salvage procedures, the conventional approach is to use systemic treatment with androgen deprivation, or chemohormonal therapy, usually considered as palliative treatment (20). However, with increasing advances in molecular imaging, such as cholinebased and more recently PSMA-ligand PET/CT, small-volume metastatic disease to lymph nodes can be detected with great accuracy, prompting clinicians to undertake local salvage treatments using radiation or SLND. When surgery for nodal recurrence is being selected, a bilateral ePLND adhering to the standard primary ePLND templates is most commonly advised (20).

Molecular imaging can localize lymph node metastases before surgery and, as such, provide a road map to guide sLND or salvage radiotherapy. Several series based mainly on choline PET have suggested an oncologic benefit in selected patients (21-31). The increasing implementation of sLND has raised intense discussions on the extent of the template that should be treated locally. It clearly depends on the extent of prior PLND, the accessibility of anatomic areas, and the localization of suspected lymph node metastases on preoperative imaging.

Even with a detailed surgical road map provided by PSMAligand PET, reliable intraoperative identification and removal of metastatic lymph nodes are challenging because these lesions might be atypically located, small, or morphologically unrecognizable. Intraoperative guidance is of great value. To cover applications in image-guided surgery, new PSMA tracer compounds have been and are still being developed. The first clinical implementation of such tracers can be found in PSMA-radioguided surgery (Fig. 2A) (32-34). On the basis of PSMA-directed DOTA-conjugated EuK (=Glu-urea-Lys)-based inhibitors, the DOTAGA-conjugate PSMA-I\&T (imaging and therapy) has been developed and optimized for increased PSMA affinity and in vivo stability $(33,35)$. In this respect, this DOTAGA-based ligand proved advantageous because it forms stable complexes with a broad variety of radiometals (36). Initially, ${ }^{111}$ In (half-life, $2.8 \mathrm{~d} ; \gamma$-energy, 173 and $245 \mathrm{keV}$ ) was chosen in previously developed PSMA-I\&T ligands as a $\gamma$-emitter for radioguided surgery (33). In biodistribution studies on LNCaP xenograft-bearing mice, ${ }^{111}$ In-PSMA-I\&T showed a further reduction of hepatobiliary excretion and almost exclusive renal excretion due to its reduced lipophilicity, as well as demonstrating a slightly reduced background signal but comparable tumor 

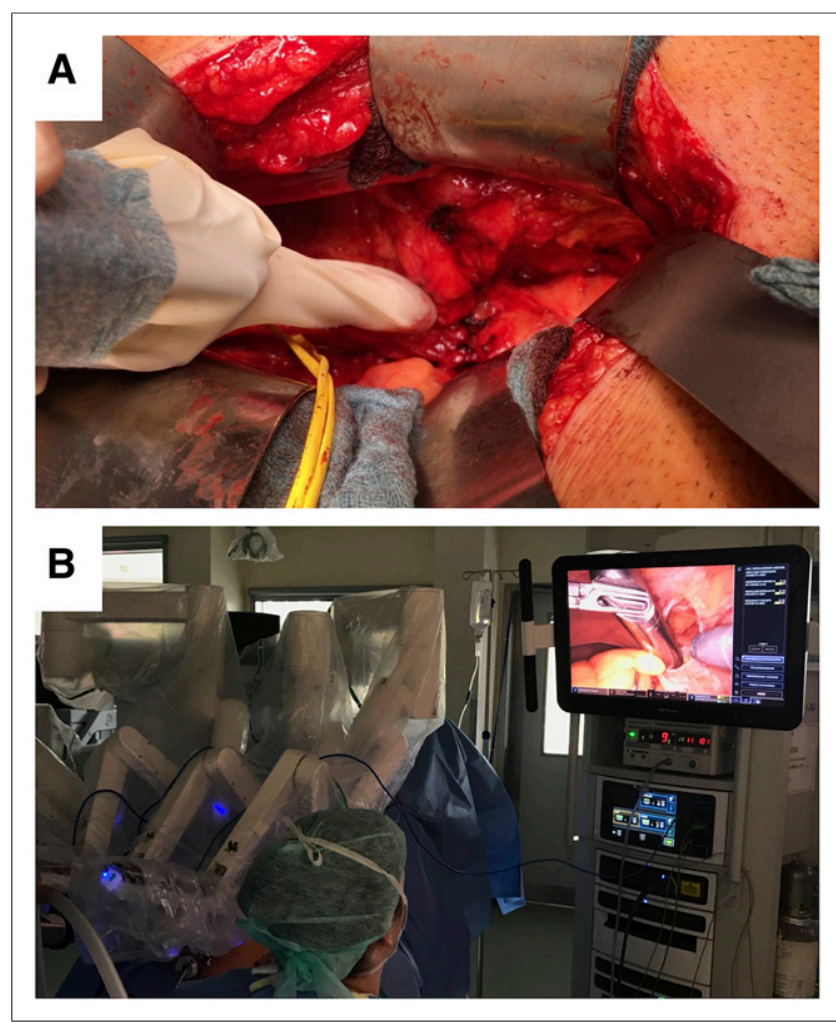

FIGURE 2. (A) Conventional rigid wireless y-probe for open PSMAradioguided surgery. (B) Drop-in y-probe for minimally invasive robotic PSMA-radioguided surgery.

uptake translating into superior tumor-to-background ratios (33). Further refinement of PSMA-targeting tracers for radioguided surgery were triggered by the high radiation exposure and restricted availability of ${ }^{111} \mathrm{InCl}_{3}$ and its high costs. Thus, the concept was adapted to allow for ${ }^{99 \mathrm{~m}} \mathrm{Tc}$-labeling because ${ }^{99 \mathrm{~m}} \mathrm{Tc}$ represents an inexpensive, readily available alternative radionuclide used as a standard in nuclear medicine and radioguided surgery (34). Compared with ${ }^{111}$ In-PSMA-I\&T, the newly developed ${ }^{99 m}$ Tc-mas3-ynal-k(Sub-KuE) ( ${ }^{99 m}$ Tc-PSMA-I\&S [imaging and surgery]) showed delayed clearance kinetics due to high plasma protein binding (94\%) but identical uptake in PSMA-positive tissues $1 \mathrm{~h}$ after injection in a LNCaP xenograft model. After at least $5 \mathrm{~h}$ after injection, excellent lesion-to-background ratios were observed due to the synergistic effect of persistent ${ }^{99 m}$ Tc-PSMA-I\&S uptake and continuing clearance of background activity, which compared favorably with the performance of ${ }^{111}$ In-PSMA-I\&T. These characteristics led to replacement of the earlier ${ }^{111}$ In-PSMA-I\&T (used in 31 patients) with the currently used ${ }^{99 m}$ Tc-PSMA-I\&S as a tracer in PSMA-radioguided surgery (now used in over 250 patients) (37). After staging via PSMA-ligand PET, patients have been selected for PSMA-guided surgery in the setting of salvage surgery. Those patients who were thought to benefit from PSMAguided surgery based mainly on a low PSA value and only a few lesions seen on PSMA-ligand PET received a second intravenous injection of ${ }^{111} \mathrm{In}-\mathrm{PSMA}-\mathrm{I} \& \mathrm{~T}$ or ${ }^{99 \mathrm{~m}} \mathrm{Tc}$-PSMA-I\&S to facilitate intraoperative lesion identification $(33,34)$. After administration of the $\gamma$-emitting PSMA tracers, SPECT/CT imaging was performed to confirm concordance in tracer accumulation with preoperative PSMA-ligand PET. Because of the difference in sensitivity and spatial resolution, PSMA-ligand SPECT tended to miss small lesions (Fig. 3) (38). Intraoperatively, however, the use of a conventional $\gamma$-probe-similar to the ones used during sentinel node procedures (39) - allowed for accurate intraoperative detection of PSMA-positive lesions (Fig. 4) that can be confirmed by autoradiographic studies (40). This concept was even compatible with the forward-thinking freehand SPECT technology (32).

Recently, the introduction of a so-called drop-in $\gamma$-probe (41) has allowed for PSMA-guided surgery during robot-assisted laparoscopic surgery (Fig. 2B) (42). Intraoperatively, $\gamma$-probes not only facilitate intraoperative in vivo guidance but also enable ex vivo measurements to confirm successful resection of these metastatic PC lesions, with a specificity of more than $95 \%$ for ${ }^{99 \mathrm{~m}} \mathrm{Tc}-$ PSMA-I\&S $(32,37)$. In cases of positive identification, defined as measurements exceeding at least twice the background level of the patient's noncancerous fatty tissue, intraoperative frozen-section histopathologic analysis is no longer required to confirm successful resection of a PET-positive lesion $(37,43)$. Furthermore, adjacent resected tissues that were part of the lymph node dissection can be examined for tumor presence by $\gamma$-probe measurements. If additional lesions are detected, the planned field of dissection can be extended to increase the probability of complete resection of all tumor-bearing tissue.

In the field of image-guided surgery, next to radioguidance there is an increasing interest in fluorescence guidance. The strength of having fluorescent tracers would be their ability to provide the operating surgeon with real-time optical lesion identification (Figs. $5 \mathrm{~A}$ and $5 \mathrm{~B}$ ). The value of such optical guidance is, however, somewhat limited as a result of light attenuation by tissue; light attenuation limits detectability to less than $1 \mathrm{~cm}$ deep in tissue and impacts the detection sensitivity (44). Although most fluorescence-based PSMA-targeting concepts are still confined to the preclinical stage, there is an ongoing clinical trial in the United Kingdom using a fluorophore-conjugated reagent (45). Preliminary data suggest that the fluorescence approach is feasible and highly likely to impact future care, with the increasing compatibility of multicolor fluorescence imaging with state-of-the-art
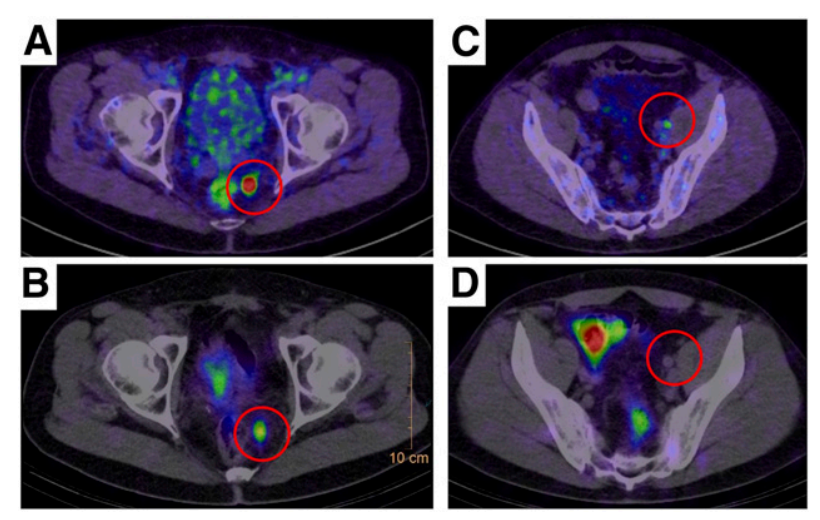

FIGURE 3. Preoperative PSMA-ligand PET/CT fusion images ( $A$ and $C$ ) and corresponding preoperative ${ }^{99 m T C-P S M A-I \& S ~ S P E C T / C T ~(B ~ a n d ~ D) ~}$ of patients who underwent PSMA-radioguided surgery with histologic confirmation of lymph node metastasis. Whereas larger lymph nodes on PSMA-ligand PET/CT (e.g., perirectal lymph node in A) can also be detected on preoperative ${ }^{99 \mathrm{~m} T C-P S M A-l i g a n d ~ S P E C T / C T ~(B), ~ s m a l l e r ~}$ lymph nodes on PSMA-ligand PET/CT (e.g., iliac lymph node in C) might not be detectable by preoperative ${ }^{99 m T c-P S M A-l i g a n d ~ S P E C T / C T ~(D) . ~}$ 

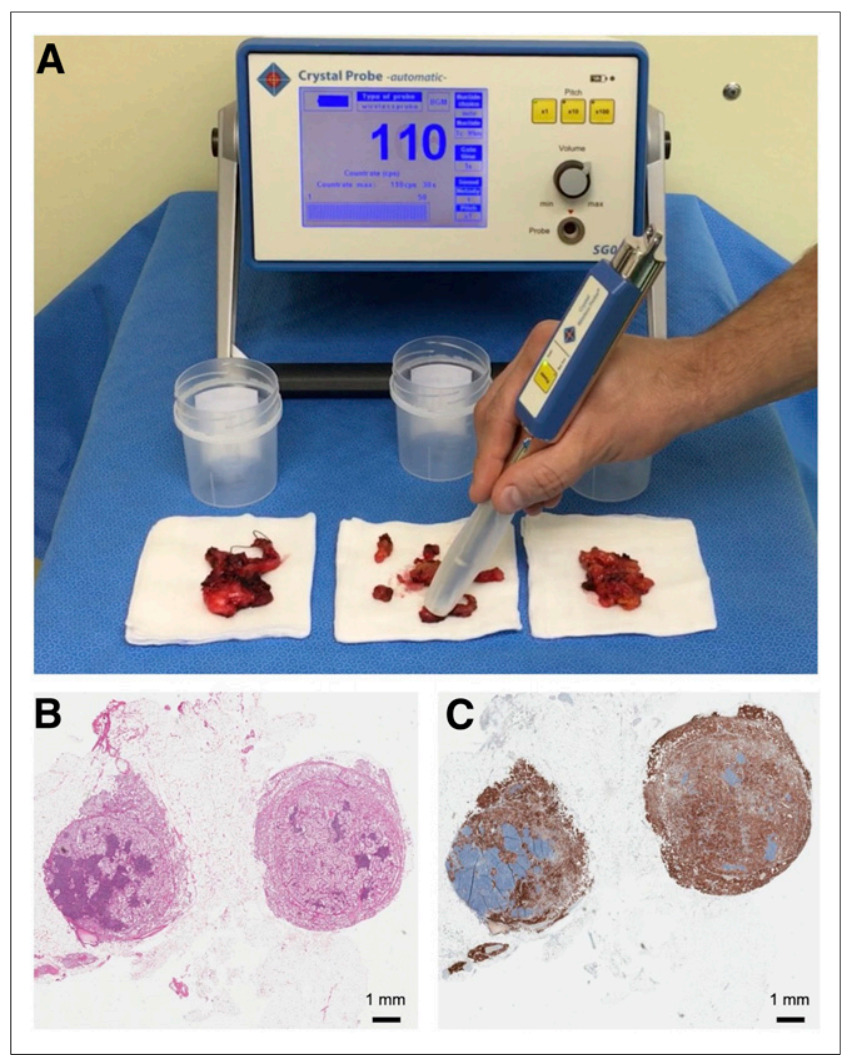

FIGURE 4. (A) Ex vivo measurements with y-probe during PSMAradioguided surgery. ( $B$ and $C$ ) Histologic examination reveals lymph node metastasis in hematoxylin- and eosin-stained sections (B) and in PSMA immunohistochemistry sections (C).

laparoscopic and robotic surgical platforms $(46,47)$. To combine the benefits of the radioguided and fluorescence-guided surgery concept, bimodal or, rather, hybrid tracers have been introduced into the clinic-for example, for sentinel node imaging in PC patients (48). The clinical impact of this work has also sparked the development of hybrid tracers for PSMA-targeted applications (Fig. 5C). Examples of small-molecule hybrid PSMA tracers under investigation in the preclinical arena are ${ }^{111} \mathrm{In}$-LICOR-800CW-LysDOTA-EuK (49), ${ }^{68} \mathrm{Ga}-\mathrm{NIR}$ 800CW-PSMA-11 (50), ${ }^{68} \mathrm{Ga}$-PSMA $\mathrm{I} \& \mathrm{~F}(51)$, and ${ }^{99 \mathrm{~m}} \mathrm{Tc}-\mathrm{EuK}-\left(\mathrm{SO}_{3}\right) \mathrm{Cy} 5-\mathrm{mas}_{3}$ (52). These promising hybrid initiatives are further extended with ongoing efforts that explore Cerenkov imaging of ${ }^{68} \mathrm{Ga}-\mathrm{PSMA}-11$ (Fig. 5D) (53).

\section{COMPARISON OF PSMA-GUIDED SLND TO STANDARD APPROACHES}

Currently, PSMA-guided surgery has been used mostly in recurrent PC. The main goal of metastasis-directed therapy in recurrent $\mathrm{PC}$ is to delay cancer progression and limit the associated toxicity of systemic palliative therapy (e.g., androgen deprivation) (31). With the advancements of molecular imaging and especially since the introduction of PSMA-ligand PET, targeted salvage therapies such as sLND or salvage radiotherapy, although still considered experimental by guidelines, are increasingly sought after for patients with minimal recurrent disease (54). However, diverging short-term outcomes after standard sLND based on PSMA-ligand PET have been reported, with rates of complete biochemical response (postoperative PSA $<0.2 \mathrm{ng} / \mathrm{mL}$ ) ranging between $19 \%$ and $59 \%(21,25,29,55,56)$. Some authors have even concluded that SLND is not appropriate either to cure disease or even to delay further systemic treatment (55). These seemingly contradictory results might be due to patient characteristics causing a different likelihood of harboring systemic disease (23). To estimate the risk and to counsel patients about sLND or salvage radiotherapy, several clinical variables can be considered in addition to the findings of PSMA-ligand PET imaging.

A recent large multiinstitutional analysis evaluated significant predictors of early clinical recurrence $(<12 \mathrm{mo})$ after sLND (25). Multivariate analysis suggests that a higher Gleason grade group, a shorter time from radical prostatectomy to rising PSA values, concurrent antiandrogen deprivation therapy, and higher absolute PSA values, besides a higher number of lesions on PET and a retroperitoneal location, are significant predictors of early clinical recurrence after sLND (25). On the other hand, poor outcomes could also be caused by incomplete removal of metastatic lesions (55). After previous ePLND or salvage radiotherapy, the surgical field might be complicated by fibrotic changes. Small metastatic lymph nodes could also be located deep in the pelvis and outside the usual field of dissection. Thus, especially in sLND, guidance using molecularly targeted approaches such as PSMA-radioguided surgery has created much interest in the urologic and nuclear medicine community $(32,37,43,57,58)$.

In the largest series (121 patients) undergoing PSMA-radioguided surgery, metastatic lesions could be removed from almost all patients $(99 \%)$, leading to a complete biochemical response in $66 \%$ of patients. Low PSA level and a single lesion on PSMAligand PET were associated with a higher likelihood of complete biochemical response (84\%) and longer biochemical recurrencefree survival (14 mo). However, microscopic spread to neighboring lymph nodes cannot be detected using either PSMA-ligand PET or PSMA-radioguided surgery. Although the extent of sLND using PSMA-radioguided surgery (or sLND in general) is currently under intensive discussion, treating only the suspected lymph node

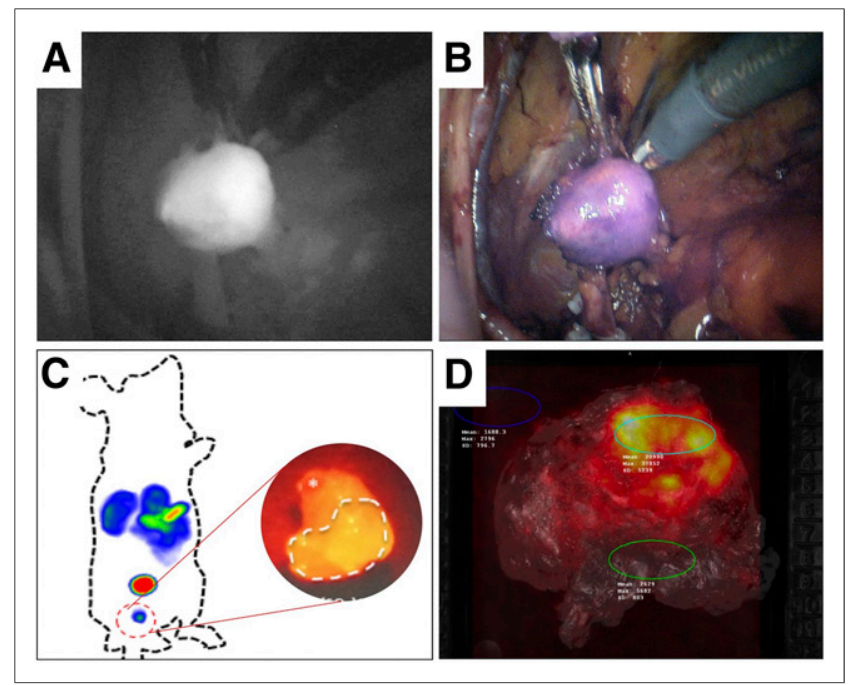

FIGURE 5. Examples of novel optical and hybrid PSMA applications. (A and B) Clinical application of fluorescent PSMA tracer-guided dissection of lymph node metastasis during robotic surgery. (C) Preclinical application of hybrid ${ }^{99 \mathrm{~m} T c-E u K-}\left(\mathrm{SO}_{3}\right) \mathrm{Cy} 5-\mathrm{mas}_{3}$ PSMA-ligand combining radioguided and fluorescence-guided surgery (52). (D) Cerenkov imaging of radical prostatectomy specimen by use of ${ }^{8} \mathrm{Ga}$-PSMA-11 (53). 
on PSMA-ligand PET is not advisable. Conversely, sLND procedures should be accompanied by at least a clean dissection of the respective side. Still, emerging data seem to hint at the superiority of PSMA-radioguided salvage surgery over standard sLND (59). The main drawbacks for analysis and comparisons of sLND series are not only the differences in study populations and the lack of long-term follow-up in most studies but also the differences in evaluated endpoints or definitions of progression (23).

External-beam radiotherapy is another treatment option for locoregional recurrence or oligometastatic disease. However, the comparison of sLND series to salvage radiotherapy is impeded even more by the different characteristics of treated patient cohorts. Although most patients who undergo sLND do not harbor metastatic disease (M1) by definition, but instead present with molecular imaging-classified locoregional lymph node metastases, patients undergoing metastasis-directed therapy by radiation often show oligometastatic bone lesions and thus represent truly M1 patients and, consequently, a more advanced disease state $(31,60)$. Nevertheless, although oncologic outcome data are still awaited, it is tempting to believe that SLND might provide benefits for locoregional lymph node metastases in properly selected patients because it shows a good safety profile, yields histologic proof, and allows radiotherapy as an additional treatment option.

In summary, PSMA-ligand PET enables early detection of metastatic lesions in recurrent $\mathrm{PC}$ and is currently recommended for this indication by the guidelines of the European Association of Urology (20). In selected and well-informed patients, sLND can be discussed as an experimental approach on the basis of clinical characteristics and PSMA-ligand PET imaging results but should ideally be undertaken only in the context of well-conducted clinical trials. In addition, PSMA-guided procedures might further enable precise intraoperative identification and removal of metastatic lesions and might thus be a useful adjunct to sLND. Clinical trials to investigate the oncologic benefits of sLND and the benefit of a management change based on the results of PSMA-ligand PET are now warranted.

\section{COMPARISON OF PSMA-GUIDED PRIMARY LYMPH NODE DISSECTION TO STANDARD APPROACHES}

In primary PC, the goal of primary lymph node dissection is twofold: to stage disease status accurately and to achieve a potential cure in a selected group of men with node-positive disease. However, there is no prospective evidence that ePLND benefits oncologic outcomes (61-66). This lack of evidence, combined with the perceived increased morbidity of the added procedure, is driving a decline in the practice of lymphadenectomy, except in the presence of high-grade or high-volume disease. Current guidelines recommend treatment of the lymphatic drainage at the time of radiotherapy or radical prostatectomy if the risk of nodal metastasis exceeds $5 \%$ by commonly used nomograms such as the Briganti, Partin, and Memorial Sloan Kettering Cancer Center nomograms (usually representing intermediate- and highrisk PC) (67-69). Guidelines also recommend that primary PC patients with a higher risk profile should not be spared ePLND irrespective of negative findings on imaging (20). Because lymphatic drainage of primary PC is diverse, and most involved lymph nodes are in the internal, external, and common iliac fields, as well as in the obturator fossa lymph node template fields, an ePLND is recommended at the time of surgery $(20,70)$. Thus, although ePLND has not yet been shown to improve oncologic outcome, it currently represents the method of choice for correct staging and prognosis (26).

On the downside, ePLND might be associated with potential complications, further reinforcing the importance of precision surgery strategies that aide nodal dissections. These approaches need to accommodate both micrometastases and macrometastases. Because the first draining lymph nodes in some patients might be outside the usual proposed ePLND template, sentinel approaches targeting the lymph node have been proposed and have already shown additional value for correct lymph node staging and reduction of morbidity $(71,72)$. In a recent systematic review, different lymphatic mapping techniques have shown a high sensitivity of $95.2 \%$ and a high negative predictive value of $98.0 \%$, compared with ePLND, and seem to improve the nodal yield when combined with ePLND $(20,73)$.

For identification of macrometastases, PSMA-ligand PET seems to be the method of choice and could influence clinical management by, for example, extending the dissection of regular ePLND in cases of out-of-template lymph node metastases. Although PSMAligand PET shows higher sensitivity than conventional imaging, it still falls short for detection of small metastatic lesions, hindering its use to preclude patients without evidence of nodal disease from ePLND. Thus, for identification of micrometastases in the lymphatics, one still needs to revert to the traditional lymphatic mapping approaches (72).

Will PSMA-guided surgery impact clinical care in the primary treatment of PC? In theory, it could be used in primary high-risk, high-volume PC patients with evidence of lymph node-positive disease on PSMA-ligand PET (32). However, there is a likelihood that additional small lymph node metastases are present in primary PC but not detected by PSMA-ligand PET, and clinical parameters (e.g., PSA value) are not as helpful as in recurrent PC. Thus, ePLND is still advisable in patients with PSMA-ligand PET-positive lymph nodes (if considered for surgery), and the additional value of PSMA-radioguided surgery might be evident only in atypically located lesions that are not readily accessible.

\section{FUTURE PERSPECTIVES}

The concept of molecularly targeted PSMA-guided surgery was first developed in-and is being increasingly used to detect metastatic lymph nodes in-the salvage setting. As more data on oncologic short- and long-term follow-up emerge, this technology can be expected to spread and be offered at an increasing number of specialized centers during SLND procedures. Careful selection of patients with locoregional recurrence based on PSMA-ligand PET and suitable clinical characteristics is mandatory (57). With the development of drop-in $\gamma$-probes for robot-assisted minimally invasive surgery, patient discomfort and morbidity are likely to decrease, fueling further demand for such molecularly targeted approaches to achieve effective precision surgery with improved outcomes (41,42). Modifications and further developments of PSMA-directed tracers, especially in combination with fluorescence, will enable visual or even bimodal tracking by fluorescence cameras and $\gamma$-probes $(46,47,49,50)$. Tools for advanced intraoperative navigation via augmented-reality and virtual reality displays may further help to increase the surgical targeting accuracy while reducing morbidity (74).

Currently, there is also great interest in translating PSMAtargeted surgical approaches to the primary setting - in the context of lymph node dissection or even during margin assessment of the 
prostate during radical prostatectomy. In patients with suggestive lymph nodes on PSMA-ligand PET, combining ePLND with intraoperative PSMA guidance could be helpful, especially when the involved lymph nodes are outside the standard template. However, the likelihood of underestimation of lymphatic spread by PSMAligand PET, especially at primary diagnosis, has to be considered. Furthermore, all currently available tracers for PSMA-radioguided surgery show urinary excretion and urinary spillage from the bladder during radical prostatectomy, and such spillage can contaminate the field for PSMA-guided lymph node dissection (75). Development of tracers with less or even no renal elimination is desirable. As for margin assessment, urinary excretion for current available tracers is an even greater challenge as compared to lymph node dissection. Moreover, when the prostatic fossa is being evaluated after prostatectomy to detect the presence of residual cancer cells, precision is impeded by physiologic tracer accumulation within the rectal wall background, compounded by weak signals from the few and dispersed cancer cells. In these clinical scenarios, development of tracers without renal elimination and with less unspecific accumulation in the rectal wall is desirable. When the prostatectomy specimen is being evaluated for positive surgical margins, the tissue penetration of $\gamma$-rays precludes the use of current PSMA tracers for radioguided surgery because only tumor cells on the surface of the positive resection margin are of interest. A clear unmet need is the development of tracers that do not penetrate healthy tissue layers and are not excreted in the urine. Optical surgical guidance could also help provide a possible solution to this problem.

Over the past decade, major advances have been achieved in image-guided surgery for PC. With advancing technologies, new tracers, and optical engineering, it is likely that these advances will soon have an impact on management of the disease. Further evaluations of these technologies are warranted through wellconducted clinical trials to provide robust evidence of overall effectiveness, cost effectiveness, and reproducibility of outcomes. Finally, experiences in the field of PSMA-guided surgery may serve as an exemplar for the future development and use of similar molecularly guided approaches on other malignancies and diseases (76).

\section{DISCLOSURE}

Hans-Jürgen Wester is the chief executive officer of Scintomics $\mathrm{GmbH}$ and owns stock or options in the company. Fijs van Leeuwen is the chief innovation officer at ORSI Academy. No other potential conflict of interest relevant to this article was reported.

\section{REFERENCES}

1. Mazzone E, Preisser F, Nazzani S, et al. Location of metastases in contemporary prostate cancer patients affects cancer-specific mortality. Clin Genitourin Cancer. 2018;16:376-384.e1.

2. van Leeuwen FWB, Winter A, van der Poel HG, et al. Technologies for imageguided surgery for managing lymphatic metastases in prostate cancer. Nat Rev Urol. 2019;16:159-171.

3. Meijer HJ, Fortuin AS, van Lin EN, et al. Geographical distribution of lymph node metastases on MR lymphography in prostate cancer patients. Radiother Oncol. 2013;106:59-63.

4. Leijte JA, Valdes Olmos RA, Nieweg OE, Horenblas S. Anatomical mapping of lymphatic drainage in penile carcinoma with SPECT-CT: implications for the extent of inguinal lymph node dissection. Eur Urol. 2008;54:885-890.

5. Afshar-Oromieh A, Zechmann CM, Malcher A, et al. Comparison of PET imaging with a ${ }^{68} \mathrm{Ga}$-labelled PSMA ligand and ${ }^{18} \mathrm{~F}$-choline-based PET/CT for the diagnosis of recurrent prostate cancer. Eur J Nucl Med Mol Imaging. 2014;41:11-20.

6. Morigi JJ, Stricker PD, van Leeuwen PJ, et al. Prospective comparison of ${ }^{18} \mathrm{~F}$ fluoromethylcholine versus ${ }^{68} \mathrm{Ga}$-PSMA PET/CT in prostate cancer patients who have rising PSA after curative treatment and are being considered for targeted therapy. J Nucl Med. 2015;56:1185-1190.

7. Moghul M, Somani B, Lane T, et al. Detection rates of recurrent prostate cancer: ${ }^{68}$ gallium (Ga)-labelled prostate-specific membrane antigen versus choline PET/ CT scans-a systematic review. Ther Adv Urol. 2019;11:1756287218815793.

8. Silver DA, Pellicer I, Fair WR, Heston WD, Cordon-Cardo C. Prostate-specific membrane antigen expression in normal and malignant human tissues. Clin Cancer Res. 1997;3:81-85.

9. Maurer T, Eiber M, Schwaiger M, Gschwend JE. Current use of PSMA-PET in prostate cancer management. Nat Rev Urol. 2016;13:226-235.

10. Eiber M, Fendler WP, Rowe SP, et al. Prostate-specific membrane antigen ligands for imaging and therapy. J Nucl Med. 2017;58(suppl):67S-76S.

11. Afshar-Oromieh A, Avtzi E, Giesel FL, et al. The diagnostic value of PET/CT imaging with the ${ }^{68} \mathrm{Ga}$-labelled PSMA ligand HBED-CC in the diagnosis of recurrent prostate cancer. Eur J Nucl Med Mol Imaging. 2015;42:197-209.

12. Eiber M, Maurer T, Souvatzoglou M, et al. Evaluation of hybrid ${ }^{68} \mathrm{Ga}-\mathrm{PSMA}$ ligand PET/CT in 248 patients with biochemical recurrence after radical prostatectomy. J Nucl Med. 2015;56:668-674.

13. Afshar-Oromieh A, Holland-Letz T, Giesel FL, et al. Diagnostic performance of ${ }^{68}$ Ga-PSMA-11 (HBED-CC) PET/CT in patients with recurrent prostate cancer: evaluation in 1007 patients. Eur J Nucl Med Mol Imaging. 2017;44:1258-1268.

14. Fendler WP, Calais J, Eiber M, et al. Assessment of ${ }^{68}$ Ga-PSMA-11 PET accuracy in localizing recurrent prostate cancer: a prospective single-arm clinical trial. JAMA Oncol. 2019;5:856-863.

15. Rauscher I, Duwel C, Haller B, et al. Efficacy, predictive factors, and prediction nomograms for ${ }^{68} \mathrm{Ga}$-labeled prostate-specific membrane antigen-ligand positronemission tomography/computed tomography in early biochemical recurrent prostate cancer after radical prostatectomy. Eur Urol. 2018;73:656-661.

16. Maurer T, Gschwend JE, Rauscher I, et al. Diagnostic efficacy of ${ }^{68}$ galliumPSMA positron emission tomography compared to conventional imaging for lymph node staging of 130 consecutive patients with intermediate to high risk prostate cancer. J Urol. 2016;195:1436-1443.

17. Jilg CA, Rischke HC, Beck T, et al. Diagnostic accuracy of Ga-68-HBED-CCPSMA-ligand-PET/CT before salvage lymph node dissection for recurrent prostate cancer. Theranostics. 2017;7:1770-1780.

18. Hövels AM, Heesakkers RA, Adang EM, et al. The diagnostic accuracy of CT and MRI in the staging of pelvic lymph nodes in patients with prostate cancer: a meta-analysis. Clin Radiol. 2008;63:387-395.

19. De Visschere PJL, Standaert C, Futterer JJ, et al. A systematic review on the role of imaging in early recurrent prostate cancer. Eur Urol Oncol. 2019;2:47-76.

20. Mottet N, van den Bergh RCN, Briers E, et al. Prostate cancer. European Association of Urology website. https://uroweb.org/guideline/prostate-cancer/. Accessed November 22, 2019.

21. Suardi N, Gandaglia G, Gallina A, et al. Long-term outcomes of salvage lymph node dissection for clinically recurrent prostate cancer: results of a single-institution series with a minimum follow-up of 5 years. Eur Urol. 2015;67:299-309.

22. Jilg CA, Leifert A, Schnell D, et al. Toxicity and quality of life after cholinePET/CT directed salvage lymph node dissection and adjuvant radiotherapy in nodal recurrent prostate cancer. Radiat Oncol. 2014;9:178.

23. Ploussard G, Gandaglia G, Borgmann H, et al. Salvage lymph node dissection for nodal recurrent prostate cancer: a systematic review. Eur Urol. 2019;76:493-504.

24. Karnes RJ, Murphy CR, Bergstralh EJ, et al. Salvage lymph node dissection for prostate cancer nodal recurrence detected by ${ }^{11} \mathrm{C}$-choline positron emission tomography/computerized tomography. J Urol. 2015;193:111-116.

25. Fossati N, Suardi N, Gandaglia G, et al. Identifying the optimal candidate for salvage lymph node dissection for nodal recurrence of prostate cancer: results from a large, multi-institutional analysis. Eur Urol. 2019;75:176-183.

26. Bandini M, Fossati N, Briganti A. Salvage surgery for nodal recurrent prostate cancer. Curr Opin Urol. 2017;27:604-611.

27. Tilki D, Mandel P, Seeliger F, et al. Salvage lymph node dissection for nodal recurrence of prostate cancer after radical prostatectomy. J Urol. 2015;193:484-490.

28. Porres D, Pfister D, Thissen A, et al. The role of salvage extended lymph node dissection in patients with rising PSA and PET/CT scan detected nodal recurrence of prostate cancer. Prostate Cancer Prostatic Dis. 2017;20:85-92.

29. Herlemann A, Kretschmer A, Buchner A, et al. Salvage lymph node dissection after ${ }^{68} \mathrm{Ga}$-PSMA or ${ }^{18} \mathrm{~F}-\mathrm{FEC}$ PET/CT for nodal recurrence in prostate cancer patients. Oncotarget. 2017;8:84180-84192.

30. Steuber T, Jilg C, Tennstedt P, et al. Standard of care versus metastases-directed therapy for PET-detected nodal oligorecurrent prostate cancer following multimodality treatment: a multi-institutional case-control study. Eur Urol Focus. 2019; 5:1007-1013.

31. Ost P, Reynders D, Decaestecker K, et al. Surveillance or metastasis-directed therapy for oligometastatic prostate cancer recurrence: a prospective, randomized, multicenter phase II trial. J Clin Oncol. 2018;36:446-453. 
32. Maurer T, Weirich G, Schottelius M, et al. Prostate-specific membrane antigenradioguided surgery for metastatic lymph nodes in prostate cancer. Eur Urol. 2015;68:530-534.

33. Schottelius M, Wirtz M, Eiber M, Maurer T, Wester HJ. [ ${ }^{111}$ In]PSMA-I\&T: expanding the spectrum of PSMA-I\&T applications towards SPECT and radioguided surgery. EJNMMI Res. 2015;5:68.

34. Robu S, Schottelius M, Eiber M, et al. Preclinical evaluation and first patient application of ${ }^{99 \mathrm{~m} T c-P S M A-I \& S}$ for SPECT imaging and radioguided surgery in prostate cancer. J Nucl Med. 2017;58:235-242.

35. Banerjee SR, Pullambhatla M, Byun Y, et al. ${ }^{68} \mathrm{Ga}$-labeled inhibitors of prostatespecific membrane antigen (PSMA) for imaging prostate cancer. J Med Chem. 2010;53:5333-5341.

36. Weineisen M, Schottelius M, Simecek J, et al. ${ }^{68} \mathrm{Ga}$ - and ${ }^{177} \mathrm{Lu}$-labeled PSMA I\&T: optimization of a PSMA-targeted theranostic concept and first proof-ofconcept human studies. J Nucl Med. 2015;56:1169-1176.

37. Maurer T, Robu S, Schottelius M, et al. ${ }^{99 \mathrm{~m}}$ Tc-based PSMA-radioguided surgery in recurrent prostate cancer. Eur Urol. 2019;75:659-666.

38. Rauscher I, Maurer T, Souvatzoglou M, et al. Intrapatient comparison of ${ }^{111} \mathrm{In}-$ PSMA I\&T SPECT/CT and hybrid ${ }^{68} \mathrm{Ga}$-HBED-CC PSMA PET in patients with early recurrent prostate cancer. Clin Nucl Med. 2016;41:e397-e402.

39. Van Oosterom MN, Rietbergen DDD, Welling MM, Van der Poel HG, Maurer T, van Leeuwen FWB. Recent advances in nuclear and hybrid detection modalities for image-guided surgery. Expert Rev Med Devices. 2019;16:711-734.

40. Maurer T, Schwamborn K, Schottelius M, et al. PSMA theranostics using PET and subsequent radioguided surgery in recurrent prostate cancer. Clin Genitourin Cancer. 2016;14:e549-e552.

41. Meershoek P, van Oosterom MN, Simon H, et al. Robot-assisted laparoscopic surgery using DROP-IN radioguidance: first-in-human translation. Eur J Nucl Med Mol Imaging. 2019;46:49-53.

42. van Leeuwen FWB, van Oosterom MN, Meershoek P, et al. Minimal-invasive robot-assisted image-guided resection of prostate-specific membrane antigenpositive lymph nodes in recurrent prostate cancer. Clin Nucl Med. 2019;44: 580-581.

43. Rauscher I, Duwel C, Wirtz M, et al. Value of ${ }^{111}$ In-prostate-specific membrane antigen (PSMA)-radioguided surgery for salvage lymphadenectomy in recurrent prostate cancer: correlation with histopathology and clinical follow-up. BJU Int. 2017;120:40-47.

44. KleinJan GH, Bunschoten A, van den Berg NS, et al. Fluorescence guided surgery and tracer-dose, fact or fiction? Eur J Nucl Med Mol Imaging. 2016;43:1857-1867.

45. Prostate molecular targeting to enhance surgery using IR800 IAB2M. ISRCTN Registry website. https://www.isrctn.com/editorial/retrieveFile/bb913a29-f155435a-8d21-921fed454fed/34933. Updated March 27, 2019. Accessed November $22,2019$.

46. van den Berg NS, Buckle T. KleinJan GH, van der Poel HG, van Leeuwen FWB. Multispectral fluorescence imaging during robot-assisted laparoscopic sentinel node biopsy: a first step towards a fluorescence-based anatomic roadmap. Eur Urol. 2017;72:110-117.

47. Meershoek P, KleinJan GH, van Oosterom MN, et al. Multispectral-fluorescence imaging as a tool to separate healthy from disease-related lymphatic anatomy during robot-assisted laparoscopy. J Nucl Med. 2018;59:1757-1760.

48. van Leeuwen FWB, Schottelius M, Brouwer OR, et al. Trending: radioactive and fluorescent bimodal/hybrid tracers as multiplexing solutions for surgical guidance. J Nucl Med. November 11, 2019 [Epub ahead of print].

49. Banerjee SR, Foss CA, Horhota A, et al. ${ }^{111} \mathrm{In}$ - and IRDye800CW-labeled PLAPEG nanoparticle for imaging prostate-specific membrane antigen-expressing tissues. Biomacromolecules. 2017;18:201-209.

50. Baranski AC, Schafer M, Bauder-Wust U, et al. PSMA-11-derived dual-labeled PSMA inhibitors for preoperative PET imaging and precise fluorescence-guided surgery of prostate cancer. J Nucl Med. 2018;59:639-645.

51. Schottelius M, Wurzer A, Wissmiller K, et al. Synthesis and preclinical characterization of the PSMA-targeted hybrid tracer PSMA-I\&F for nuclear and fluorescence imaging of prostate cancer. $J$ Nucl Med. 2019;60:71-78.

52. Hensbergen AW, Buckle T, van Willigen DW, et al. Hybrid tracers based on cyanine backbones targeting prostate-specific membrane antigen: tuning pharmacokinetic properties and exploring dye-protein interaction. J Nucl Med. September 3, 2019 [Epub ahead of print].

53. Costa PF, Darr C, Binse I, et al. Early results of intraoperative ${ }^{68} \mathrm{Ga}$-PSMA Cerenkov luminescence imaging in radical prostatectomy [abstract]. J Nucl Med. 2019;60(suppl 1):658.

54. Aluwini SS, Mehra N, Lolkema MP, et al. Oligometastatic prostate cancer: results of a Dutch multidisciplinary consensus meeting. Eur Urol Oncol. August 7, 2019 [Epub ahead of print].
55. Hanske J, Ostholt J, Roghmann F, et al. Salvage lymph node dissection in hormone-naive men: how effective is surgery? Urol Oncol. 2019;37:812.e17812.e24.

56. Siriwardana A, Thompson J, van Leeuwen PJ, et al. Initial multicentre experience of ${ }^{68}$ gallium-PSMA PET/CT guided robot-assisted salvage lymphadenectomy: acceptable safety profile but oncological benefit appears limited. BJU Int. 2017;120:673-681.

57. Horn T, Kronke M, Rauscher I, et al. Single lesion on prostate-specific membrane antigen-ligand positron emission tomography and low prostate-specific antigen are prognostic factors for a favorable biochemical response to prostate-specific membrane antigen-targeted radioguided surgery in recurrent prostate cancer. Eur Urol. 2019;76:517-523.

58. Maurer T, van Leeuwen FWB, Schottelius M, Wester HJ, Eiber M. Entering the era of molecular-targeted precision surgery in recurrent prostate cancer. $J \mathrm{Nucl}$ Med. December 20, 2018 [Epub ahead of print].

59. Knipper S, Tilki D, Mansholt J, et al. Metastases-yield and prostate-specific antigen kinetics following salvage lymph node dissection for prostate cancer: a comparison between conventional surgical approach and prostate-specific membrane antigen-radioguided surgery. Eur Urol Focus. 2019;5:50-53.

60. Siva S, Bressel M, Murphy DG, et al. Stereotactic ablative body radiotherapy (SABR) for oligometastatic prostate cancer: a prospective clinical trial. Eur Urol. 2018;74:455-462.

61. Choo MS, Kim M, Ku JH, Kwak C, Kim HH, Jeong CW. Extended versus standard pelvic lymph node dissection in radical prostatectomy on oncological and functional outcomes: a systematic review and meta-analysis. Ann Surg Oncol. 2017;24:2047-2054.

62. Fossati N, Willemse PM, van den Broeck T, et al. The benefits and harms of different extents of lymph node dissection during radical prostatectomy for prostate cancer: a systematic review. Eur Urol. 2017;72:84-109.

63. Mandel P, Kriegmair MC, Bogdan K, et al. Association between lymph node counts and oncological outcomes in lymph node positive prostate cancer. Eur Urol Focus. 2017;3:248-255.

64. Rees T, Raison N, Sheikh MI, et al. Is extended pelvic lymph node dissection for prostate cancer the only recommended option? A systematic over-view of the literature. Turk J Urol. 2016;42:240-246.

65. Abdollah F, Gandaglia G, Suardi N, et al. More extensive pelvic lymph node dissection improves survival in patients with node-positive prostate cancer. Eur Urol. 2015;67:212-219.

66. Preisser F, van den Bergh RCN, Gandaglia G, et al. Effect of extended pelvic lymph node dissection on oncologic outcomes in D'Amico intermediate- and highrisk radical prostatectomy patients: a multi-institutional study. J Urol. August 22, 2019 [Epub ahead of print].

67. Cimino S, Reale G, Castelli T, et al. Comparison between Briganti, Partin and MSKCC tools in predicting positive lymph nodes in prostate cancer: a systematic review and meta-analysis. Scand J Urol. 2017;51:345-350.

68. Gandaglia G, Fossati N, Zaffuto E, et al. Development and internal validation of a novel model to identify the candidates for extended pelvic lymph node dissection in prostate cancer. Eur Urol. 2017;72:632-640.

69. Roach M III, Marquez C, Yuo HS, et al. Predicting the risk of lymph node involvement using the pre-treatment prostate specific antigen and Gleason score in men with clinically localized prostate cancer. Int J Radiat Oncol Biol Phys. 1994;28:33-37.

70. Heck MM, Retz M, Bandur M, et al. Topography of lymph node metastases in prostate cancer patients undergoing radical prostatectomy and extended lymphadenectomy: results of a combined molecular and histopathologic mapping study. Eur Urol. 2014;66:222-229.

71. Wit EMK, Acar C, Grivas N, et al. Sentinel node procedure in prostate cancer: a systematic review to assess diagnostic accuracy. Eur Urol. 2017;71:596-605.

72. van der Poel HG, Wit EM, Acar C, et al. Sentinel node biopsy for prostate cancer: report from a consensus panel meeting. BJU Int. 2017;120:204-211.

73. Grivas N, Wit EMK, Kuusk T, et al. The impact of adding sentinel node biopsy to extended pelvic lymph node dissection on biochemical recurrence in prostate cancer patients treated with robot-assisted radical prostatectomy. J Nucl Med. 2018;59:204-209.

74. van Oosterom MN, van der Poel HG, Navab N, van de Velde CJH, van Leeuwen FWB. Computer-assisted surgery: virtual- and augmented-reality displays for navigation during urological interventions. Curr Opin Urol. 2018;28: 205-213.

75. van Leeuwen FW, van der Poel HG. Surgical guidance in prostate cancer: "from molecule to man" translations. Clin Cancer Res. 2016;22:1304-1306.

76. Rischpler C, Schwamborn K, Schwaiger M, Maurer T. Radio-guided-surgery of a paravertebral paraganglioma using I-123-MIBG. Nuklearmedizin. 2018;57:N2-N3. 